\title{
Study on Financial Market Risk Measurement Based on GJR-GARCH and FHS
}

\author{
Hong Zhang, Jian Guo, Li Zhou \\ School of Information, Beijing Wuzi University, Beijing, China
}

Email address:

514050209@qq.com (Hong Zhang)

To cite this article:

Hong Zhang, Jian Guo, Li Zhou. Study on Financial Market Risk Measurement Based on GJR-GARCH and FHS. Science Journal of Applied Mathematics and Statistics. Vol. 3, No. 3, 2015, pp. 70-74. doi: 10.11648/j.sjams.20150303.12

\begin{abstract}
In this paper, we establish GJR-GARCH models to extract the residuals of logarithmic returns of one kind of Chinese stock index--- Shanghai Composite Index and the series of independent and identically distribution standardized residuals is formed from the filtered model residuals and conditional volatilities from the return series with an GJR-GARCH model. The results show that from the contrast of actual value and lower limit of predicted VaR value, actual index value for 8 days breaks below the prediction lower limit. The fitting result of VaR method to the market risk of the Shanghai composite index is better than that of the Traditional Historical Simulation.
\end{abstract}

Keywords: VaR, FHS, GJR-GARCH Model, Financial Market Risk

\section{Introduction}

In this paper, we establish GJR-GARCH models to extract the residuals of logarithmic returns of Shanghai Composite Index. The estimation of residual-distribution is student-t distribution whose degrees of freedom is four. Independent and identically distributed random sequence is obtained after we got the marginal distribution of the residuals of index returns and standardized residuals. Secondly, Using the residual sequence of the FHS simulation(20000 times) to calculate the var value, in different confidence level. Lastly, Comparing the var floor with closing price whose sample interval is from 2003.1.4 to 2014.12.30.

Barone Adesi (1999) ${ }^{[1,13]}$ proposed an improved historical simulation method based on FHS-GARCH model, to carry on the modelling of future assets and swap value distribution. Barone Adesi and Giannopoulos $(2001)^{[2,9,12]}$ studied the FHS method, the results show that it can make up for the deficiency of the traditional simulation method, and has carried on the comparative study with the traditional sampling method. Barone a Adesi et al $(2002)^{[3,14,15]}$ use FHS VaR calculated values for different combinations of assets, the results show that the method for the application of FHS risk management is more effective. Giannopoulos and Tunaru $(2005)^{[4,10,11]}$ study and proved FHS method is a method to calculate the expected short-loss (Expeeted short), And also got a statistical error formula for computing the model error. Pritsker $(2006)^{[5-8]}$ from the perspective of theoretical and empirical study of three historical simulation VaR method: two traditional historical simulation method and the FHS method.FHS is considered to be a promising approach, but still needs further development.

There are 3 Levelltitles in an article to make ideas clear:

(1) Obtaining independent and identically distributed random sequence.

(2) Calculating the var value through simulation.

(3) Comparing the var floor with closing price.

\section{The Empirical Analysis}

\subsection{The Selection of Data and Its Characteristics}

Selecting S.H.I (Shanghai composite index) as research object. Sample interval is from 2003.1.4 to 2014.12.30. The essential information is below.

Known from figuer 2, Distritution of daily logarithmic return of Shanghai composite index is fat-tail on both ends of gains and losses. And JB- statistic is significantly. So the results refuse the hypothesis of normal distribution. 


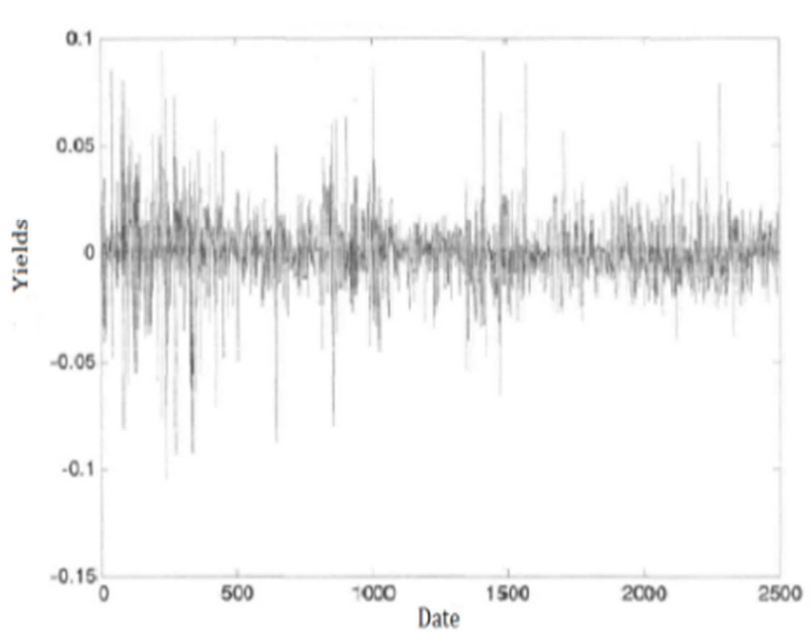

Figure 1. Daily logarithmic return of Shanghai composite index.

Table 1. Statistical indicators of Shanghai composite index.

\begin{tabular}{lll}
\hline Name & Mean & STD \\
\hline S.H.I & 0.0003 & 0.0167 \\
Slewness & Kurtosis & JB- statistic \\
-0.0701 & 9.2469 & 4052.248 \\
\hline
\end{tabular}

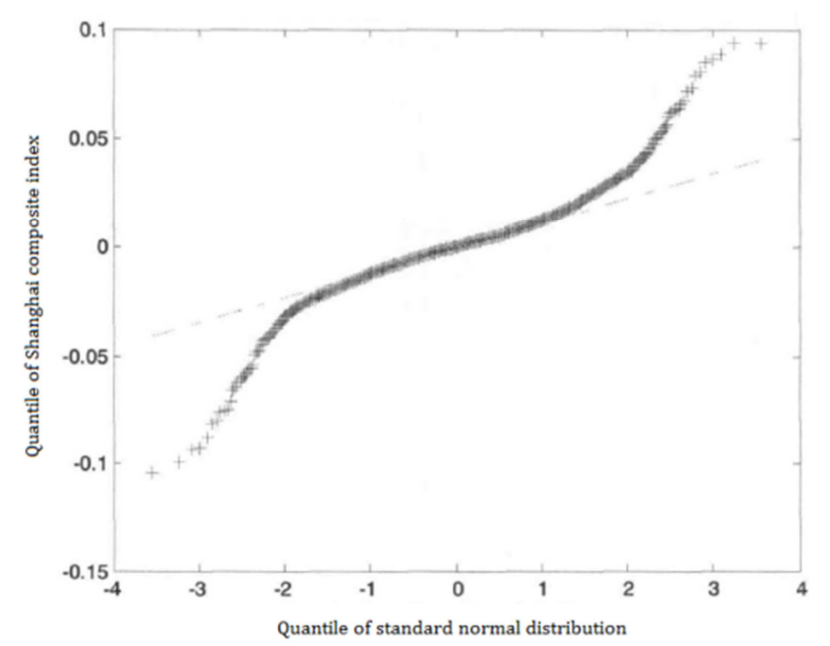

Figure 2. Normal Q-Q Plot of daily logarithmic return of Shanghai composite index.

\subsection{The Establishment of the Model and the Analysis of the Results}

Autocorrelogram and partial- autocorrelogram of the logarithmic return suggest that yield sequence presents a certain degree of serial correlation, Further, autocorrelogram of the square of the logarithmic return suggest that yield sequence square presents serial correlation significantly. (figure 3 5)

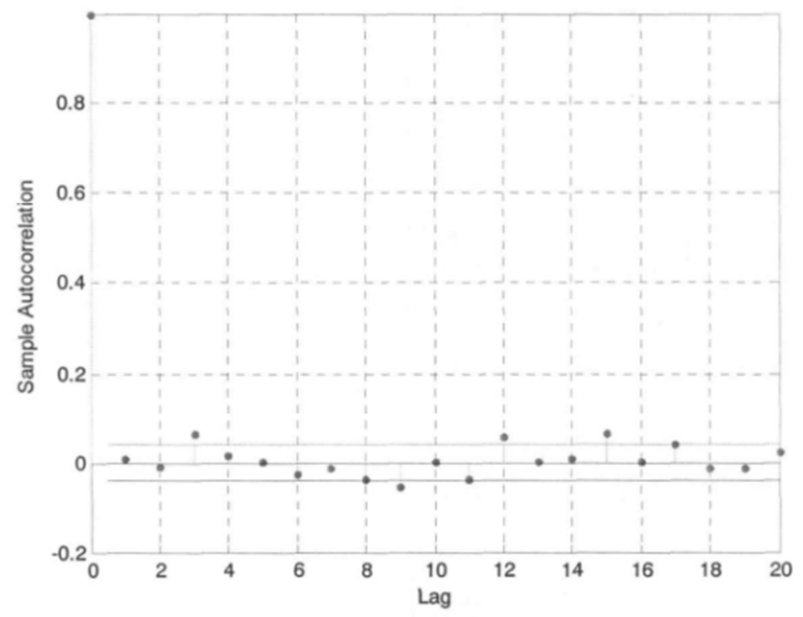

Figure 3. Autocorrelogram of logarithmic return of Shanghai composite index.

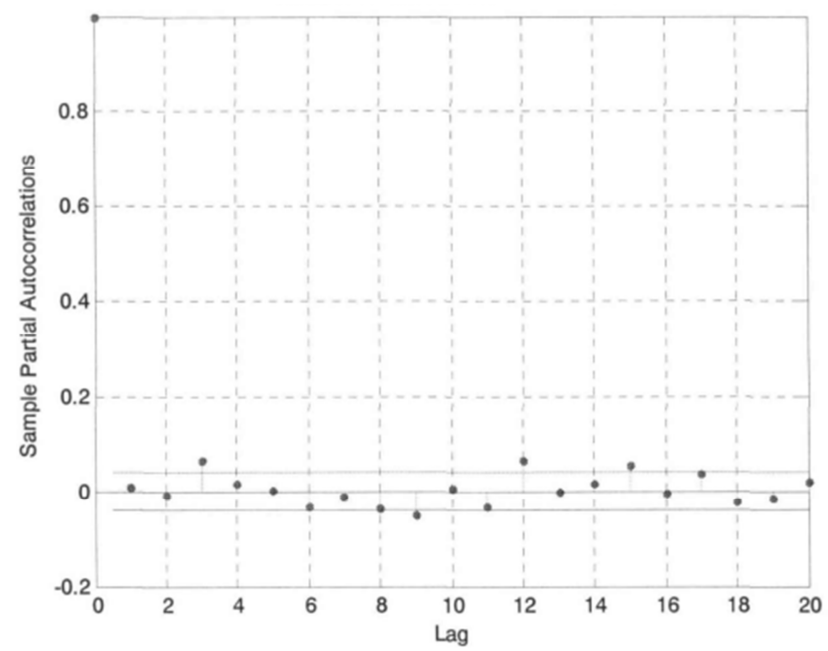

Figure 4. Partial Autocorrelogram of logarithmic return of Shanghai composite index.

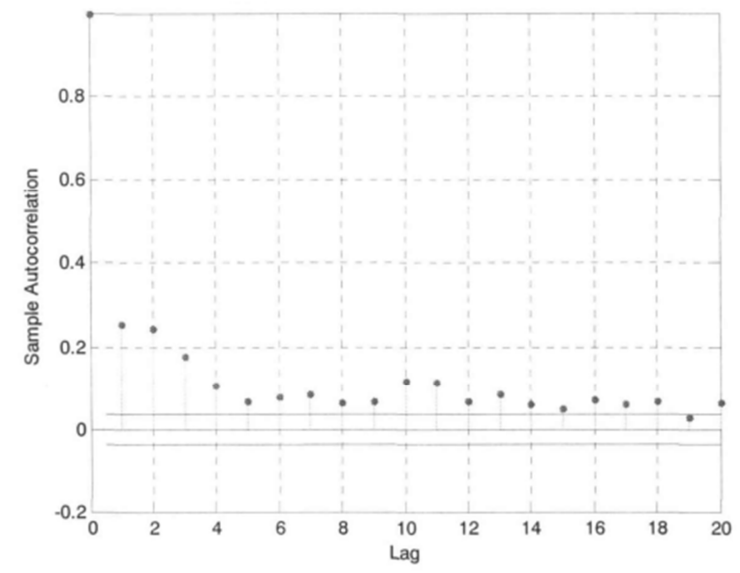

Figure 5. Autocorrelogram of logarithmic return square of Shanghai composite index.

The paper adopt Student-t distribution to estimate residuals instead of normal distribution. Next we estimate four ARMA-GJR-GARCH models. (table 2) 
Table 2. Parameters estimation\&Significant level\& Evaluation index of the models.

\begin{tabular}{|c|c|c|c|c|c|}
\hline \multirow{2}{*}{\multicolumn{2}{|c|}{ Parameters }} & \multicolumn{4}{|c|}{ The models of Shanghai composite index } \\
\hline & & Model 1 & Model 2 & Model 3 & Model 4 \\
\hline \multicolumn{2}{|l|}{$\theta_{1}$} & & & $-0.7788(0.0000)$ & $0.1526(0.0000)$ \\
\hline \multicolumn{2}{|l|}{$\theta_{2}$} & & & & $0.8161(0.0000)$ \\
\hline \multicolumn{2}{|l|}{$\theta_{3}$} & $0.07776(0.0000)$ & $0.07766(0.0000)$ & & \\
\hline \multicolumn{2}{|l|}{$\theta_{4}$} & $-0.04721(0.0012)$ & $-0.04852(0.0085)$ & & \\
\hline \multicolumn{2}{|l|}{$\eta_{1}$} & & & $0.80000(0.0000)$ & $-0.11141(0.0000)$ \\
\hline \multicolumn{2}{|l|}{$\eta_{2}$} & & & & $-0.85214(0.0000)$ \\
\hline \multicolumn{2}{|l|}{$\alpha_{1}$} & $0.08947(0.0056)$ & $0.08421(0.0001)$ & $0.00112(0.0000)$ & $0.07254(0.00004)$ \\
\hline \multicolumn{2}{|l|}{$\alpha_{2}$} & $0.02141(0.0005)$ & $-0.05482(0.0002)$ & & $0.05412(0.0001)$ \\
\hline \multicolumn{2}{|l|}{$\lambda_{1}$} & $0.05412(0.0120)$ & $0.19584(0.0000)$ & $0.07514(0.0144)$ & $0.12405(0.0018)$ \\
\hline \multicolumn{2}{|l|}{$\lambda_{2}$} & & $-0.19852(0.0007)$ & & $-0.14521(0.2541)$ \\
\hline \multicolumn{2}{|l|}{$\beta_{1}$} & $0.85412(0.0000)$ & $1.52412(0.0000)$ & $0.85412(0.0000)$ & $0.74125(0.0000)$ \\
\hline \multicolumn{2}{|l|}{$\beta_{2}$} & & $-0.52369(0.000)$ & & \\
\hline \multicolumn{2}{|l|}{ SSR } & 0.75412 & 0.76521 & 0.77120 & 0.77475 \\
\hline \multicolumn{2}{|l|}{ AIC } & -5.32145 & -5.69264 & -5.68125 & -5.21455 \\
\hline \multicolumn{2}{|l|}{ SBC } & -5.23412 & -5.67415 & -5.6666 & -5.66542 \\
\hline \multirow{3}{*}{ residual } & $\mathrm{Q}(4)$ & $6.52412(0.0374)$ & $8.45215(0.0145)$ & $11.06452(0.0040)$ & 6.00007 \\
\hline & $\mathrm{Q}(8)$ & $8.82415(0.1847)$ & $10.65475(0.1082)$ & $15.49995(0.0145)$ & $11.70004(0.0145)$ \\
\hline & $\mathrm{Q}(12)$ & $15.42253(0.1174)$ & $16.65415(0.0852)$ & $24.25411(0.0006)$ & $21.1750(0.0025)$ \\
\hline \multirow{3}{*}{$\begin{array}{l}\text { Residual } \\
\text { square }\end{array}$} & $\mathrm{Q}(4)$ & $1.96524(0.3777)$ & $0.98524(0.6325)$ & $1.36520(0.5236)$ & 0.96541 \\
\hline & $\mathrm{Q}(8)$ & $5.20122(0.5241)$ & $2.32145(0.8852)$ & $4.75821(0.5821)$ & $5.21425(0.2541)$ \\
\hline & $\mathrm{Q}(12)$ & $6.21425(0.7514)$ & $4.12585(0.9899)$ & $6.00000(0.8521)$ & $6.21452(0.6321)$ \\
\hline
\end{tabular}

According to SSR, Parameters significantly 、AIC and SBC determine the final model .The SSR of model 1 is the smallest; but Q-statistic of model 3 and model 4 both significant suggest that there are still some residual serial correlation. while Q-statistic of model 1 and model 2 are no significant, suggesting that the mean model and variance model are both great. And according to the principle of AIC and SBC, we chose the model whose AIC and SBC are minimum, that is model 1. Establishing model:

$$
r(t)=\theta_{3} r(t-3)+\theta_{6} r(t-6)+\varepsilon(t)
$$

Model 1's Asymmetric GARCH model is GJR-GARCH $(1,2)$ :

$h(t)=k+\alpha_{1} \varepsilon(t-1)^{2}+\beta_{1} h(t-1)+\lambda_{1} S_{t-1}^{-} \varepsilon(t-1)^{2}+\lambda_{2} S_{t-2}^{-} \varepsilon(t-2)^{2}$

Where

$$
\left\{\begin{array}{l}
k, \alpha_{1}, \alpha_{2}, \beta_{1}>0, \\
\alpha_{i}+\lambda_{i}>0, i=1,2 \\
S_{t-1}^{-}=\left\{\begin{array}{l}
1, \varepsilon(t-1)<0 \\
0, \varepsilon(t-1) \geq 0
\end{array}\right. \\
\beta_{1}+\sum_{j=1}^{2} \alpha_{j}+\frac{1}{2} \sum_{j=1}^{2} \lambda_{j}<1
\end{array}\right.
$$

The Seasonal ARMA model is adopted in the paper to get residual series of S.H.I; and conditional standard deviation filtering of Shanghai composite index is obtained with GJR-GARCH. (figure 6 and figure 7)

We standardize filtered residual with (3) in order to applying FHS. $(z(t)$ is the filtered standard residual at time $\mathrm{t} ; \mathrm{e}(\mathrm{t})$ is the filtered residual at $\mathrm{t}$; Conditional standard deviation of filtered residual at $t$ ). Go a step further, we analysis residual filtering of Shanghai composite index and conditional standard deviation filtering of Shanghai composite index. (figure 8 and figure 9)

The result suggests that there is no serial correlation within them; they are independent identically distributed random variables

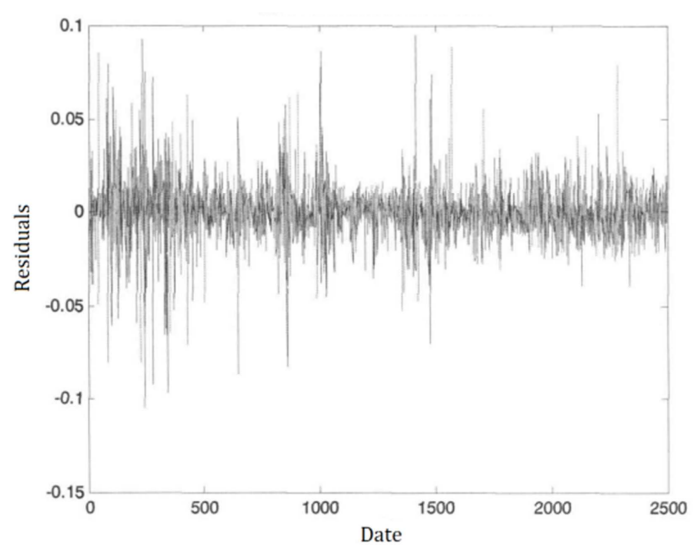

Figure 6. Residual filtering of Shanghai composite index. 


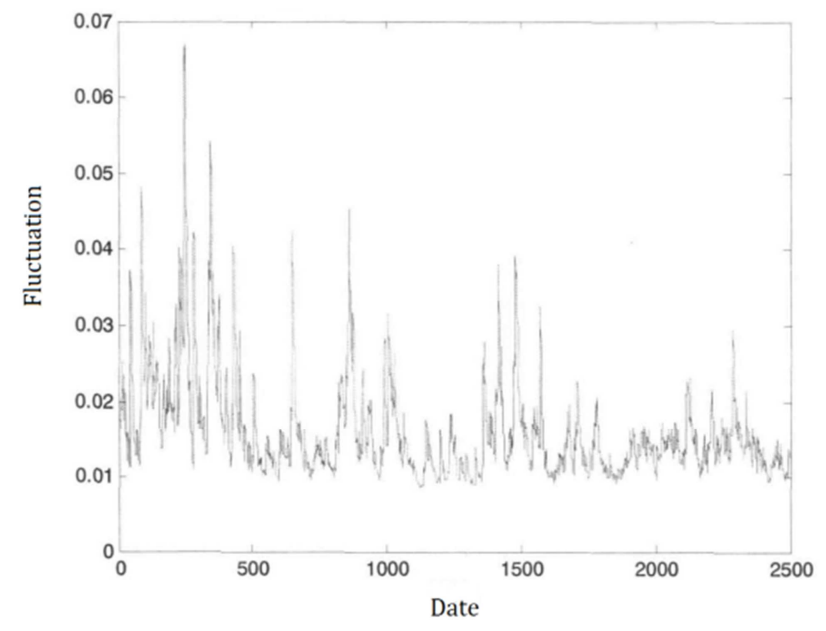

Figure 7. Conditional standard deviation filtering of Shanghai composite index.

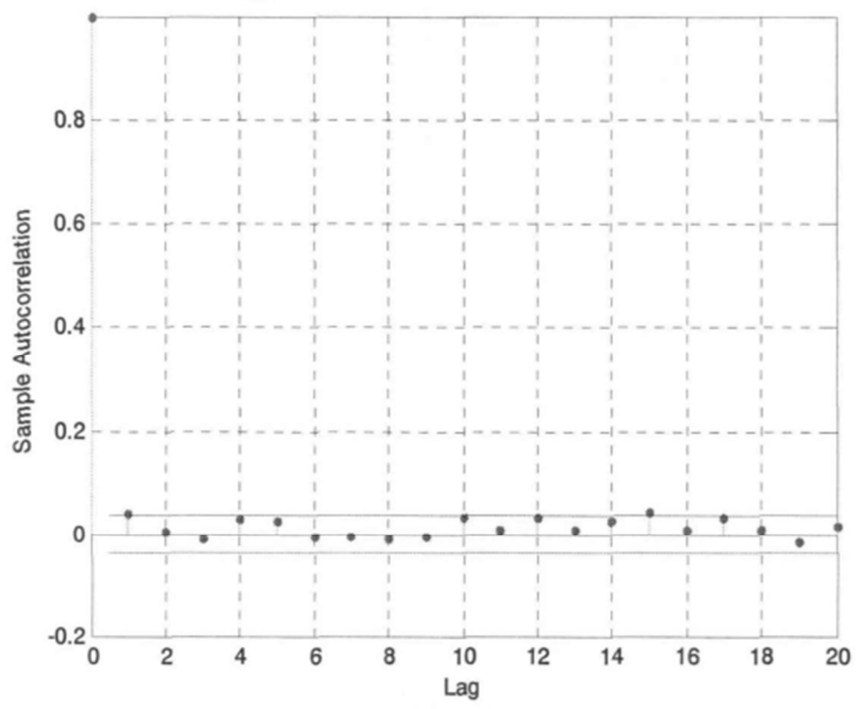

Figure 8. Autocorrelogram of standard error of Shanghai composite index.

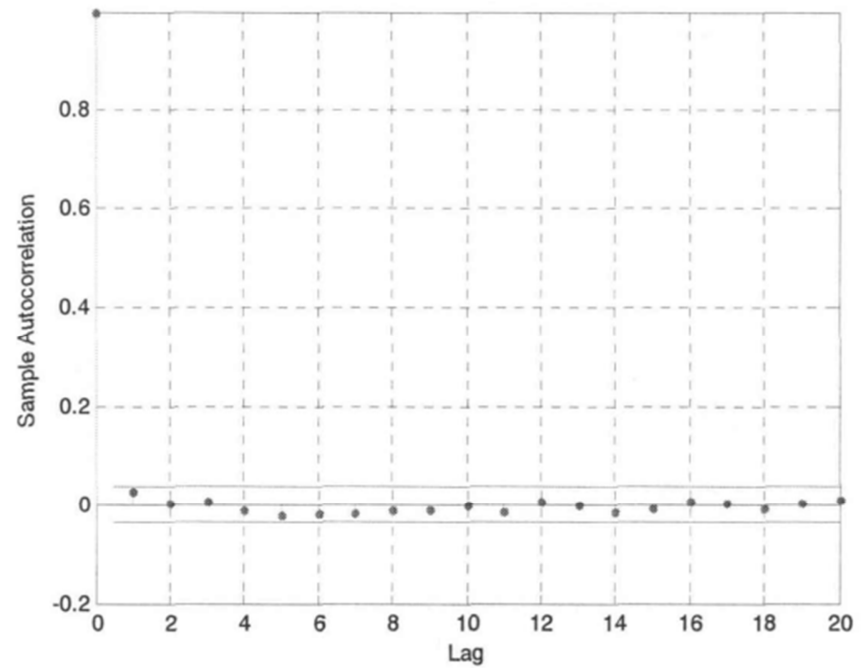

Figure 9. Autocorrelogram of standard error square of Shanghai composite index.

We sampling for 20000 times on the filtered standard residual with bootstrapping. And regard it as the input of independent identically distributed noise process of the holding period for a day of earnings forecast model.

Figure 10 is cumulative function of simulation of one day return;

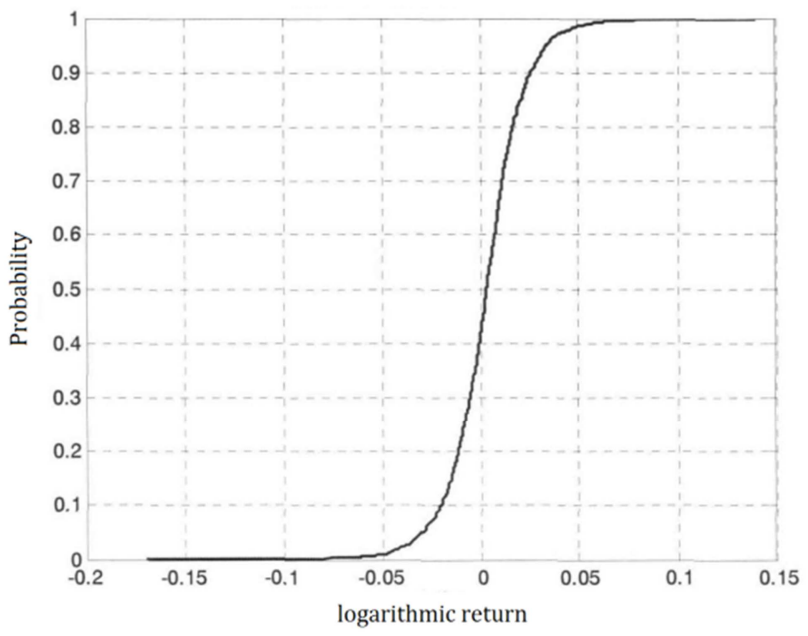

Figure 10. Cumulative function of simulation of one day return.

Figure 11 is probability density function of simulation of one day return.

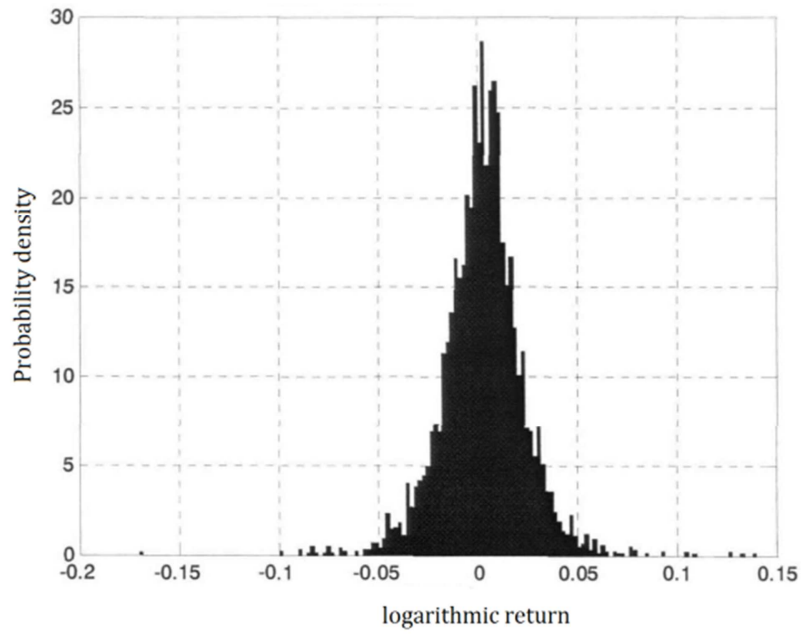

Figure 11. Probability density function of simulation of one day return.

Further, the earnings forecast is converted to the form of the closing price.

The paper chose three different confidence levels: $1 \%, 5 \%$ and $10 \%$, and calculate the VAR value in the corresponding confidence level.

In 5\% confidence level, we compare the lower limit of the predicted VAR with actual day's closing price. The results suggest that there are eight days of the actual value is less than the lower limit of the predicted VAR. Due to the confidence level that we choose is 95 , The number of days that is to say, the actual value is lower than the predicted value is not more than $240 * 5 \%=12$ days ( 240 is the number of forecast sample), while actually only 8 days. The fitting effect of VAR for this phase of the market risk is very good. Through the observation 
of historical data, when the market is relatively stable, the prediction effect of VAR is better. When the high volatility in the market, the VAR will underestimate the market risk. Therefore, in order to avoid losses of small probability event, we can consider together with the stress testing method to calculate. Obtaining a more comprehensive financial market risk assessment.

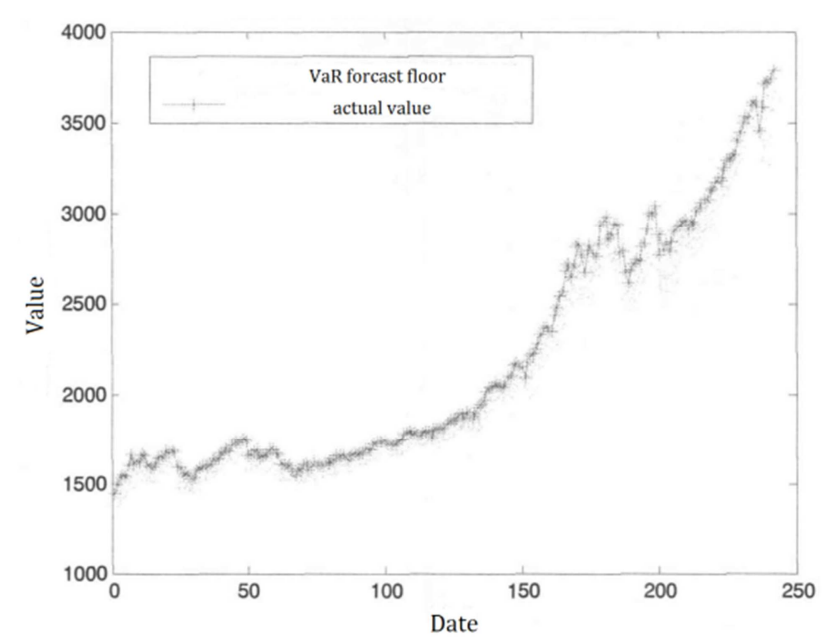

Figure 12. Trend of actual value and VaR forcast floor of S.H.I.

\section{Summary}

The fitting effect of the VAR model based on GJR of market risk is great.The results suggest that there are eight days of the actual value is less than the lower limit of the predicted VAR.Due to the confidence level that we choose is 95, The number of days that is to say, the actual value is lower than the predicted value is not more than $240 * 5 \%=12$ days ( 240 is the number of forecast sample ), while actually only 8 days. The fitting effect of VAR for this phase of the market risk is very good . Through the observation of historical data, when the market is relatively stable, the prediction effect of VAR is better. When the high volatility in the market, the VAR will underestimate the market risk. Therefore, in order to avoid losses of small probability event, we can consider together with the stress testing method to calculate. Obtaining a more comprehensive financial market risk assessment.

\section{Acknowledgements}

This project (Empirical research on Stock index investment risk model, No.68) is funded by the "2014-2015 school year, Beijing Wuzi University, College students' scientific research and entrepreneurial action plan project". And by Beijing Wuzi University, Yunhe scholars program(00610303/007). And by Beijing Wuzi University, Management science and engineering Professional group of construction projects.(No.PXM2015_014214_000039)

\section{References}

[1] Matthew Pritsker, The Hidden Dangers of Historieal Simulation, Journal of Banking\& Finance,2006(30):572-579.

[2] Kostas Giannopoulos, Radu Tunaru, Coherent risk measures under filtered historical simulation, Journal of Banking \& Finanee,2005(29):981-994.

[3] Giovanni Barone-Adesi, Kostas Giannopoulos, Les VosPer, Baektesting Derivative Portfolios with Filtered Historieal Simulation (FHS), European finaneial management, 2002 (8):33-55.

[4] Giovanni Barone-Adesi,Kostas Giannopoulos,Non-parametrie VaR Teehniques. Myths and Realities,Economic Notes, 2001(30): 169-171

[5] Giovanni Barone-Adesi,Kostas Giannopoulos, Les Vosper,VaR without Correlations for Non-Linear Portfolios,Journal of Futures Markets, 1999(19):588-601

[6] Andrey I.Kibzun, Evgeniy A.Kuznetsov.Analysis of criteria $\mathrm{VaR}$ and CVaR, Journal of Banking \& Finance,2006(30): 779-796.

[7] Malay Bhattaeharyya, Abhishek Chaudhary, Gaurav Yadav. Conditional VaR estimation using Pearson's type IV distribution European Journal of Operational Research,, 2007, in press.

[8] Allan Gregory, Jonathan Reeves. Interpreting value at risk (VaR) forecasts, 2007(3):1-20.

[9] Yau Man Zeto SamueLValue at risk and conditional extreme value theory via mark:ov regime switching models, The Journal of Futures Markets, 2008(28):155-181.

[10] Alexandra Costello, Ebenezer Asem, Eldon Gardner. Comparison of Historically Simulated VaR: Evidence from Oil Prices, Energy Economics, 2008(10):1600-1623.

[11] Malay Bhattacharyya, Gopal Ritolia. Conditional VaR using EVT Towards a planned margin scheme, International Review of Financial Analysis, 2008(17):382-395.

[12] Michael Mcaleer, Bernardo Do Veiga. Forecasting Value-at-Risk with a Parsimonious Portfolio Spillover GARCH (PS-GARCH) Model, Journal of Forecasting, 2008(27):1-19.

[13] Jenkinson A F. The frequency distribution of the annual maximum (or mimimum) values of meteorological elements, Quarterly Journal of the Royal meteorological society, 1955(81):145-158.

[14] Christian Genest,Jock MacKay. The Joy of Copulas: Bivariate Distributions with Uniform Marginals, The American Statistician, 1986(40):280-283. 